\title{
Calcaneal Osteotomy "Safe Zone" for Avoiding Injury to the Lateral Plantar Artery: A Fresh Cadaveric Study
}

Ichiro Tonogai ( $\nabla$ i.tonogai@tokushima-u.ac.jp )

Tokushima Daigaku Daigakuin Health Bioscience Kenkyubu

Koichi Sairyo

Tokushima Daigaku Daigakuin Ishiyakugaku Kenkyubu

Yoshihiro Tsuruo Tsuruo

Tokushima Daigaku Daigakuin Ishiyakugaku Kenkyubu

\section{Research}

Keywords: Lateral plantar artery, Calcaneal osteotomy, Cadaver

Posted Date: April 28th, 2020

DOl: https://doi.org/10.21203/rs.3.rs-24544/v1

License: @ (i) This work is licensed under a Creative Commons Attribution 4.0 International License. Read Full License 


\section{Abstract}

Background Calcaneal osteotomy is used to correct hindfoot deformity. Pseudoaneurysms of the lateral plantar artery (LPA) have been reported following calcaneal osteotomy and are at risk of rupture. The vascular structures in close proximity to the calcaneal osteotomy have variable courses and branching patterns. However, there is little information on the "safe zone" during calcaneal osteotomy. This study aimed to identify the safe zone that avoids LPA injury during calcaneal osteotomy.

Methods Enhanced computed tomography scans of 25 fresh cadaveric feet (13 male and 12 female specimens; mean age 79.0 years at time of death) were assessed. The specimens were injected with barium via the external iliac artery. A landmark line (line A) connecting the posterosuperior aspect of the calcaneal tuberosity and the origin of the plantar fascia was drawn and the shortest perpendicular distance between the LPA and line A was measured on sagittal images.

Results The average perpendicular distance between the LPA and line A at its closest point was $15.2 \pm 2.9 \mathrm{~mm}$. In 2 feet (8.0\%), the perpendicular distance between the LPA and line A at its closest point was very short (approximately $9 \mathrm{~mm}$ ). In 18 of the 25 feet (72.0\%), the point where the perpendicular distance from line A to the LPA was closest was the bifurcation of one of the medial calcaneal branches of the LPA, and in 7 feet ( $28.0 \%$ ) the shortest perpendicular distance from line A to the LPA was the trifurcation of the LPA, medial plantar artery, and one of the medial calcaneal branches.

Conclusion Calcaneal osteotomy performed more than $9 \mathrm{~mm}$ from line A could damage the LPA by overpenetration on the medial side. Calcaneal osteotomy on the medial side should be performed with caution to avoid iatrogenic injury to the LPA.

Level of Evidence: IV, cadaveric study

\section{Background}

Calcaneal displacement osteotomy has historically been used to correct valgus deformity of the calcaneus seen with flexible pes planovalgus deformity, rearfoot varus deformity, frontal plane deformity, sagittal plane deformity, and posterior calcaneal fractures. ${ }^{1-3}$ Calcaneal osteotomy via a lateral or oblique incision is occasionally associated with neurovascular injury, and complication rates of $5-28 \%$ have been reported in the literature. ${ }^{4-7}$

The anatomic location of the lateral plantar artery (LPA) makes it susceptible to iatrogenic injury during operative procedures involving the foot. ${ }^{8}$ One of the complications reported after calcaneal osteotomy is pseudoaneurysm of the LPA. ${ }^{9,10}$ Progressive enlargement of an untreated LPA pseudoaneurysm can compromise adjacent nerves and vessels and lead to ulceration and ultimately rupture ${ }^{11}$ if the laceration has not yet achieved full tensile strength, in addition to the fact that the area is typically subjected to continued trauma. ${ }^{12}$

Some reports have described the relationship between neurostructures and the osteotomy line ${ }^{3,13}$ and the "safe zone" for nerves during calcaneal osteotomy. ${ }^{13,14}$ Talusan et al identified a landmark line (line A) extending from the posterosuperior aspect of the calcaneal tuberosity to the origin of the plantar fascia and defined the safe zone for neural structures during calcaneal osteotomy as $11.2 \mathrm{~mm}$ anterior to line $\mathrm{A} .{ }^{14}$ However, there has been few fresh cadaveric studies of the safe zone needed to protect the LPA during calcaneal osteotomy. ${ }^{15}$

The aims of this study were to assess the shortest distance between the LPA and line A in fresh cadavers on sagittal enhanced computed tomography (CT) images and to identify a safe zone that could protect against injury to the LPA during calcaneal osteotomy.

\section{Methods}

The study was approved by our institutional review board and included 25 feet of 25 fresh cadavers (13 male and 12 female specimens; mean age 79.0 years at the time of death). Cadavers with a history or signs of ankle trauma or surgery, congenital or developmental deformity, or inflammatory arthritis were excluded.

Vessels were flushed with warm normal saline solution through a plastic catheter placed in the external iliac artery. Next, barium sulfate suspension (Barytester®, Fushimi Pharmaceutical Co, Inc, Marugame, Japan) was injected into the external iliac artery with firm manual pressure, as described in our previous reports. ${ }^{16,17}$ Enhanced multi-slice CT images (Somatom Emotion 16, Siemens Healthcare, Erlangen, Germany) of the lower extremities were obtained with the ankle in a neutral position. The sagittal images were reviewed on osseous transparent metal setting. Two specimens were dissected to observe the running of the LPA and the relationship between the LPA and the traditional Myerson osteotomy line when the osteotomy was performed $1 \mathrm{~cm}$ posterior to the posterior talar process and at a $45^{\circ}$ angle to the plantar hindfoot (posterosuperior to anteroinferior). ${ }^{18}$

The following parameters were measured in the sagittal plane: (1) the average perpendicular distance between the LPA and line A at its closest point; (2) the average distance from the posterosuperior aspect of the calcaneal tuberosity to the point on line A where LPA comes closest to line A; and (3) the average distance from the origin of the plantar fascia to the point on line A where LPA comes closest to line A (Fig. 1).

All measurements were made in triplicate by two orthopedic surgeons working independently and blinded to the purpose of the study. The values were averaged and are shown as the mean \pm standard deviation.

\section{Results}


A summary of the results is shown in Table 1, with additional details provided in Supplemental Table 1. The posterior tibial artery (PTA), LPA, and medial plantar artery (MPA) were confirmed in all specimens. In all cases, at least one of the medial calcaneal branches (arteries) crossed line A.

The average perpendicular distance between the LPA and line A at its closest point (1) was $15.2 \pm 2.9$ (range, 9.1-20.5) $\mathrm{mm}$. In 18 of the 25 feet (72.0\%), the point where the perpendicular distance from line A to the LPA was shortest was the bifurcation of one of the medial calcaneal branches from the LPA (Figure 2); in 7 feet (28.0\%), the point where the perpendicular distance from line A to the LPA was shortest was the trifurcation of the LPA, MPA, and one of the medial calcaneal branches (Figure 3). In 2 feet (8.0\%), the perpendicular distance between the LPA and line A at its closest point was approximately 9 mm (Figure 4). The average distance from the posterosuperior aspect of the calcaneal tuberosity to the point on line A where the LPA came closest to line A (2) was $26.9 \pm 5.1$ (range, 14.1-36.5) $\mathrm{mm}$, and $59.3 \%$ (range, 31.5\%-80.0\%) when the closest point along line $A$ was expressed as a percentage of the total length of line $A$ from the posterosuperior aspect of the calcaneal tuberosity. The average distance from the origin of the plantar fascia to the point on line A where the LPA came closest to line A (3) was $18.6 \pm 5.6$ (range, 8.2-30.6) $\mathrm{mm}$, and $40.7 \%$ (range, 20.0\%-68.5\%) when the closest point along line A was expressed as a percentage of the total length of line A from the posterosuperior aspect of the origin of the plantar fascia.

\section{Discussion}

The PTA is located posterior to the medial malleolus and bifurcates into the MPA and LPA. All bifurcation points of the PTA are located within the tarsal tunnel, ${ }^{19}$ while the LPA passes laterally under the proximal abductor hallucis and flexor digitorum brevis muscles, coursing along the lateral margin of the flexor digitorum brevis, where it is located superficially below the plantar fascia. The anatomic relationship of the LPA renders it vulnerable to injury during calcaneal osteotomy.

To our knowledge, this is the first cadaveric report demonstrating the distance from the LPA and line A as measured on enhanced sagittal CT images. We found that in $72.0 \%$ of cases, the point where the perpendicular distance from line A to the LPA was shortest was the bifurcation of one of the medial calcaneal branches from the LPA, and that in $28.0 \%$ of cases the point where this distance was shortest was the trifurcation of the LPA, MPA, and one of the medial calcaneal branches.

In this study, the average perpendicular distance between the LPA and line A at its closest point was $15.2 \mathrm{~mm}$, and in 2 of the 25 feet (8.0\%), the perpendicular distance between the LPA and line A at its closest point was approximately $9 \mathrm{~mm}$. Greene et al reported that the PTA crossed the Myerson osteotomy line in 2 of their 22 cases (9.1\%) when the perpendicular distance between the LPA and line A at its closest point was very short, ${ }^{15}$ as in 2 cases in our series. Therefore, there is a risk of injury to the LPA when a Myerson calcaneal osteotomy (see Fig. 5) is performed more than 9 mm anterior to line A and overpenetrates on the medial side of the calcaneus. Our findings also indicate that an anteriorly oriented osteotomy places the artery in greater proximity to the osteotomy line than would be the case with an osteotomy that is directed posteriorly, which is consistent with a report by Bruce et al. ${ }^{20}$

Consistent with findings reported by both DiDomenico et al and Greene et al that both the LPA and PTA and their branches could cross the Myerson osteotomy line ${ }^{3,15}$ we found that at least one of the medial calcaneal branches crossed line A in all cases. These observations indicate that the medial calcaneal branches (arteries) are at risk during calcaneal osteotomy. Therefore, medial calcaneal arteries could be present even within this apparent safe zone for LPA. Like Doty et al and Greene et al, we suggest that calcaneal osteotomy should be completed through the medial calcaneal cortex in a carefully controlled manner because of the proximity of vascular structures. ${ }^{9,15}$

In this study, the average closest point between line A and the LPA was 59.3\% when expressed as a percentage of the total distance of line A from the posterosuperior aspect of the calcaneal tuberosity. Greene et al reported that the average closest perpendicular point to the PTA or its branches along the Myerson osteotomy line was $57 \%$ of the total distance from the posterosuperior aspect of the calcaneal tuberosity. ${ }^{15}$ Our findings was consistent with those of Greene et al, despite the fact that we examined enhanced CT images along line A in whole fresh cadavers while they examined the Myerson osteotomy line in dissected fresh cadavers.

This study has several limitations, first is the small number of specimens used, which is inevitable due to the restricted availability of fresh-frozen cadavers in Japan. The second limitation was our acquisition of images in the neutral ankle position, considering that vascular structures vary in location with movement of the ankle. The third was a limitation in terms of the relationship between the arteries and nerves, since calcaneal osteotomy can also cause damage to nerves.

\section{Conclusions}

This study demonstrated the shortest perpendicular distance from the LPA to line A on sagittal enhanced CT images. This is the first study in which enhanced CT has been used to identify the safe zone for the LPA during calcaneal osteotomy in fresh cadaveric feet. Calcaneal osteotomy approximately more than $9 \mathrm{~mm}$ anterior to line A could injure the LPA if there is overpenetration of the medial cortex. Calcaneal osteotomy should thus be performed cautiously and with great care.

\section{Declarations}

\section{Authors' contributions}

IT was responsible for this study, and managed this study. IT performed enhanced CT examinations for fresh cadavers. IT did not result to measurement. Dr. YT measured parameters in obtained images, and supervised the enhanced CT examinations of fresh cadavers by IT. Dr. KS also measured parameters in obtained images. All authors read and approved the final manuscript. 
Ethics approval and consent to participate

This study was approved by the research board of our institution.

\section{Consent for publication}

Not applicable.

\section{Availability of data and material}

All data generated or analyzed during this study are included in this published article and its supplementary information files.

\section{Conflict of interest}

No benefits in any form have been received or will be received from a commercial party related directly or indirectly to the subject of this article.

\section{Funding}

The authors received no financial support for the research, authorship, and/or publication of this article.

\section{References}

1. Hansen ST. Surgical principles. In: Hansen ST, editor. Functional reconstruction of the foot and ankle. pp. 80. Hagerstown: Lippincott, Williams \& Wilkins; 2000. 112, 370-2.

2. Malerba F, De Marchi F. Calcaneal osteotomies. Foot Ankle Clin. 2005;10(3):523-40.

3. DiDomenico LA, Anain J, Wargo-Dorsey M. Assessment of medial and lateral neurovascular structures after percutaneous posterior calcaneal displacement osteotomy: a cadaver study. J Foot Ankle Surg. 2011;50:668-71.

4. Eastwood A. Lateral approaches in the heel. A comparison of two incisions for the fixation of calcaneal fractures. Foot. 1992;2(3):143-7.

5. Abbrasian A, Zaidi R, Guha A, Goldberg A, Cullen N, Singh D. Comparison of three different fixation methods of calcaneal osteotomies. Foot Ankle Int. 2013;34(3):420-5.

6. Kendal AR, Khalid A, Ball T, Rogers M, Cooke P, Sharp R. Complications of minimally invasive calcaneal osteotomy versus open osteotomy. Foot Ankle Int. 2015;36(6):685-90.

7. Gutteck N, Zeh A, Wohlrab D, Delank KS. Comparative results of percutaneous calcaneal osteotomy in correction of hindfoot Deformities. Foot Ankle Int. 2019;40(3):276-81. doi: 10.1177/1071100718809449. Epub 2018 Nov 9.

8. Sundararajan SR, Rajagopalakrishnan R, Rajasekaran S. Ruptured pseudoaneurysm of the lateral plantar artery after tibiotalocalcaneal fusion with retrograde nail-A rare complication. J Foot Ankle Surg. 2018;57(2):393-5. doi:10.1053/j.jfas.2017.08.017. Epub 2017 Dec 19.

9. Doty JF, Alvarez RG, Asbury BS, Rudd JN, Baxter WB. Arteriovenous fistula and pseudoaneurysm of the posterior tibial artery after calcaneal slide osteotomy: a case report. Foot Ankle Int. 2010;31(4):329-32. doi:10.3113/FAl.2010.0329.

10. Ptaszek A, Aminian A, Schneider J, Milos S. Lateral plantar artery pseudoaneurysm after calcaneal osteotomy: a case report. Foot Ankle Int. 2006;27:1413.

11. Mardjetko SM, Lubicky JP, Kuo KN, Smrcina C. Pseudoaneurysm after foot surgery. J Pediatr Orthop. 1991;11:657-62.

12. Economou PR, Paton R, Galasko CS. Traumatic pseudoaneurys of the lateral plantar artery in a child. J Pediatr Surg. 1993;28:626.

13. Durston A, Bahoo R, Kadambande S, Hariharan K, Mason L. Minimally invasive calcaneal osteotomy: does the Shannon burr endanger the neurovascular structures? A adaveric study. J Foot Ankle Surg. 2015;54(6):1062-6.

14. Talusan PG, Cata E, Tan EW, Parks BG, Guyton GP. Safe zone for neural structures in medial displacement calcaneal osteotomy: A cadaveric and radiographic investigation. Foot Ankle Int. 2015 Dec;36(12):1493-8.

15. Greene DL, Thompson MC, Gesink DS, Graves SC. Anatomic study of the medial neurovascular structures in relation to calcaneal osteotomy. Foot Ankle Int. 2001;22(7):569-71.

16. 10.1186/s13047-018-0300-3

Tonogai I, Hayashi F, Tsuruo Y, Sairyo K. Distances from the deep plantar arch to the lesser metatarsals at risk during osteotomy: a fresh cadaveric study. J Foot Ankle Res. 2018;11:57. doi: 10.1186/s13047-018-0300-3. eCollection 2018.

17. $10.1186 / \mathrm{s} 13047-018-0288-8$

Tonogai I, Hayashi F, Tsuruo Y, Sairyo K. Entry points of nutrient arteries at risk during osteotomy of the lesser metatarsals: a fresh cadaveric study. $J$ Foot Ankle Res. 2018;11:46. doi: 10.1186/s13047-018-0288-8. eCollection 2018. 
18. Myerson MS. Adult acquired flatfoot deformity: treatment of dysfunction of the posterior tibial tendon. Instr Course Lect. 1997;46:393-405.

19. Yang Y, Du ML, Fu YS, et al. Fine dissection of the tarsal tunnel in 60 cases. Sci Rep. 2017;7:46351. doi:10.1038/srep46351.

20. Bruce BG, Bariteau JT, Evangelista PE, et al. The effect of medial and lateral calcaneal osteotomies on the tarsal tunnel. Foot Ankle Int. 2014;35(4):383-8.

\section{Table 1}

Table 1. Measurements obtained for the study parameters in the cadaveric specimens.

\begin{tabular}{|c|c|c|c|c|c|c|c|}
\hline $\begin{array}{l}\text { Specimens, } \\
\mathrm{n}\end{array}$ & Sex & Side & \begin{tabular}{|l} 
Mean age \\
at death \\
(years)
\end{tabular} & $\begin{array}{l}\text { (1) Mean perpendicular distance between the LPA } \\
\text { and line A at its closest point (mm) }\end{array}$ & $\begin{array}{l}\text { (2) Mean distance } \\
\text { from the posterosuperior aspect of the calcaneal } \\
\text { tuberosity to the point on line A where the LPA } \\
\text { comes closest to line A (mm; expressed as a } \\
\text { percentage of the total length of line A) }\end{array}$ & $\begin{array}{l}\text { (3) Mean distance } \\
\text { from the origin of } \\
\text { the plantar fascia } \\
\text { to the point on } \\
\text { line A } \\
\text { where the LPA } \\
\text { comes closest to } \\
\text { line A } \\
\text { (mm; expressed } \\
\text { as a percentage } \\
\text { of the total length } \\
\text { of line A) }\end{array}$ & $\begin{array}{l}\text { Point } \\
\text { where the perpendicula } \\
\text { distance from line A } \\
\text { to the LPA is closest }\end{array}$ \\
\hline $\mathrm{n}=25$ & Male, 13 ; female, 12 & Right, 13; left, 12 & $\begin{array}{l}79.0 \pm \\
12.0\end{array}$ & $15.2 \pm 2.9$ & $26.9 \pm 5.1(59.3)$ & $18.6 \pm 5.6(40.7)$ & $\begin{array}{l}\begin{array}{l}\text { Bifurcation of one } \\
\text { of the medial calcaneal } \\
\text { branches } \\
\text { from the LPA, } n= \\
18 ; \text { trifurcation } \\
\text { of the LPA, } \\
\text { MPA, and one } \\
\text { of the medial calcaneal } \\
\text { branches, } n=7\end{array}\end{array}$ \\
\hline
\end{tabular}

LPA, lateral plantar artery; MPA, medial plantar artery

\section{Figures}

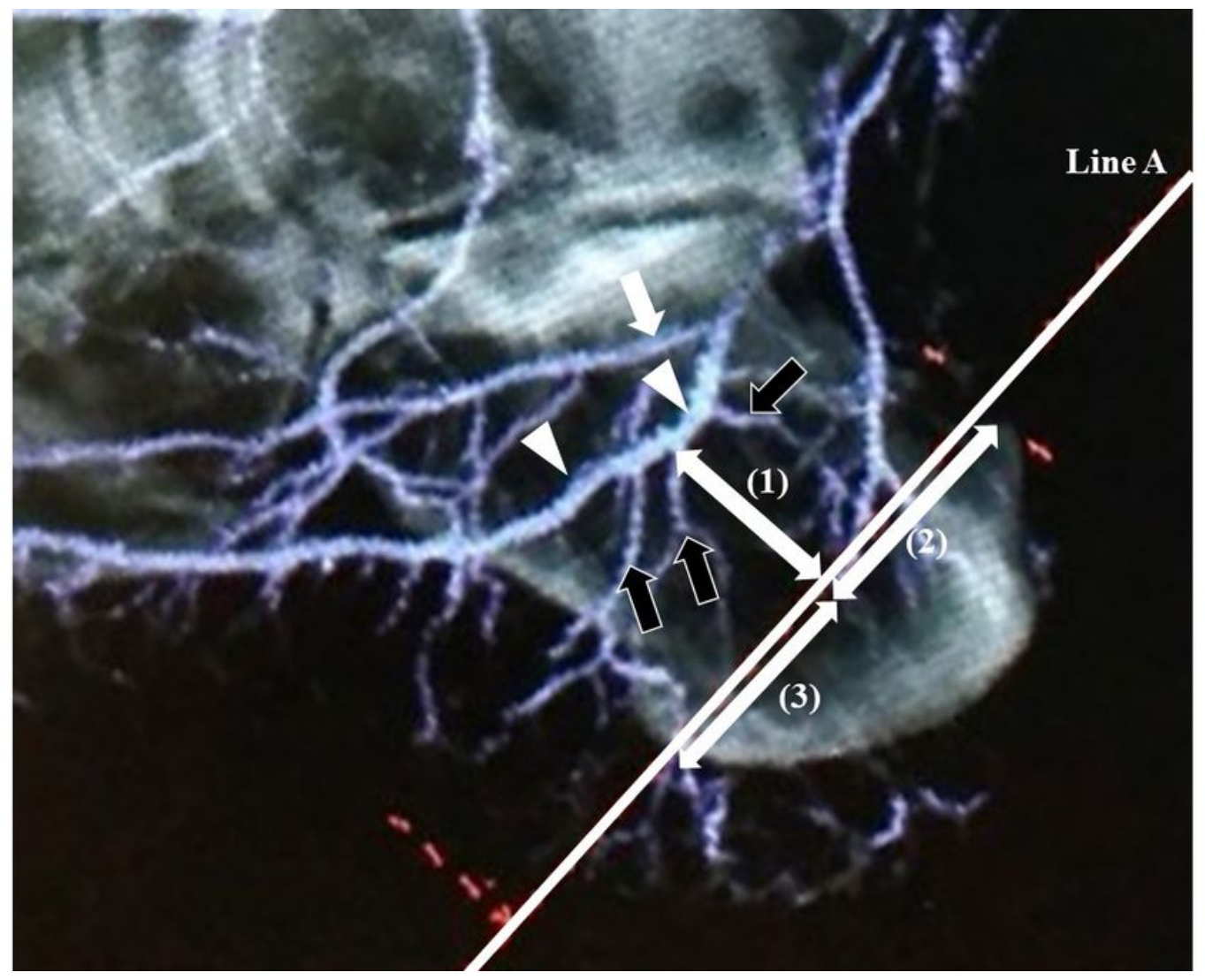

Figure 1

Enhanced computed tomography image showing the following three parameters: (1) the average perpendicular distance between the lateral plantar artery and line $A^{*}$ at its closest point; (2) the average distance from the posterosuperior aspect of the calcaneal tuberosity to the point on line $A$ where the lateral plantar artery comes closest to line A; and (3) the average distance from the origin of the plantar fascia to the point on line A where the lateral plantar artery comes closest to line A. The white arrowhead, black arrow, and white arrow indicate the lateral plantar artery, medial calcaneal branches (arteries), and medial plantar artery, respectively. *Line $A$ is the landmark line extending from the posterosuperior aspect of the calcaneal tuberosity to the origin of the plantar fascia. 


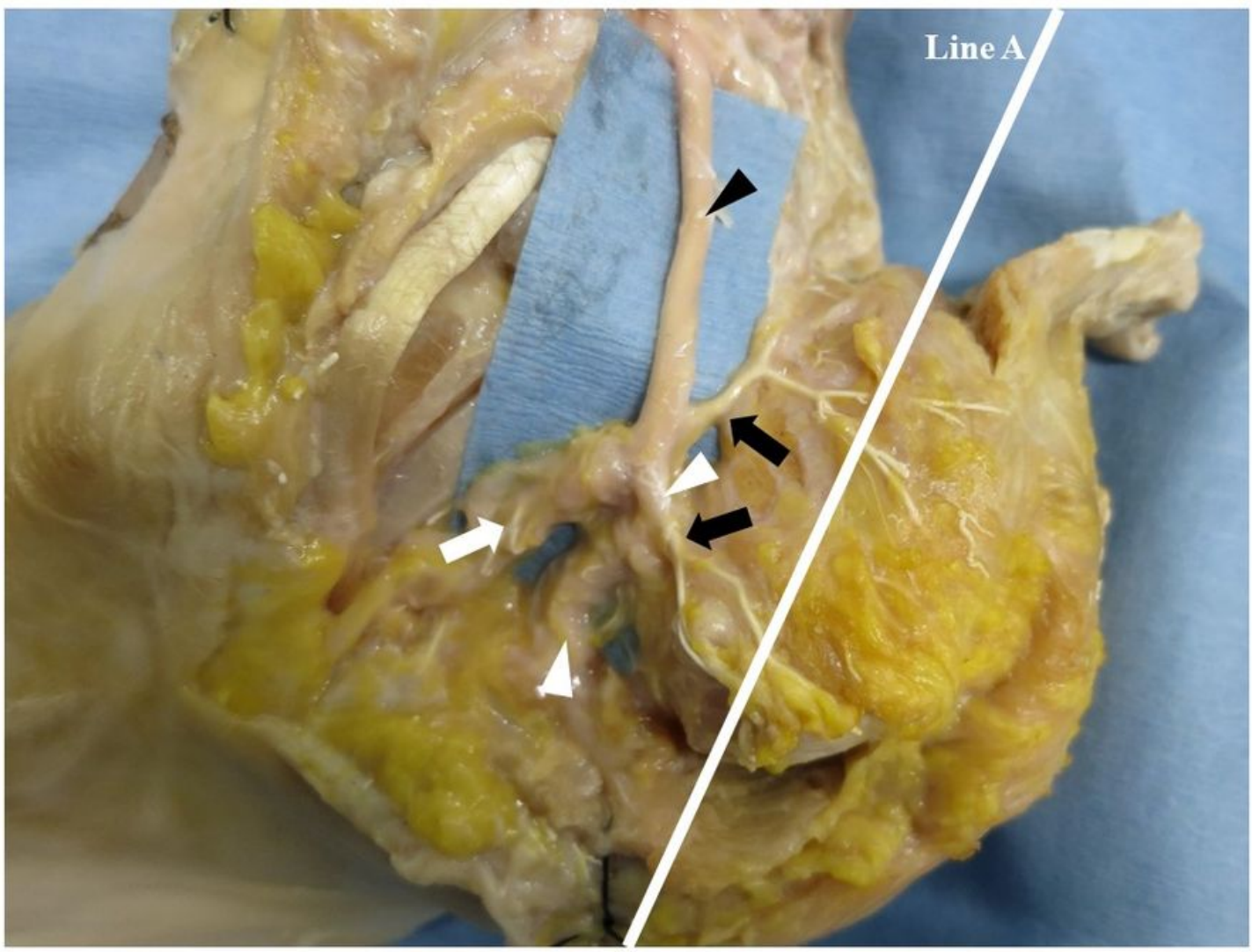

Figure 3

Photograph showing the dissected posterior tibial artery, lateral plantar artery, and medial plantar artery from the medial aspect. The tibial nerve, medial plantar nerve, lateral plantar nerve, and medial calcaneal branches (nerves) were dissected. The photograph shows that the point where the perpendicular distance from line A to the lateral plantar artery is shortest is the bifurcation of one of the medial calcaneal branches from the lateral plantar artery. The black arrowhead, white arrowhead, black arrow, and white arrow indicate the posterior tibial artery, lateral plantar artery, medial calcaneal branches (arteries), and medial plantar artery, respectively.

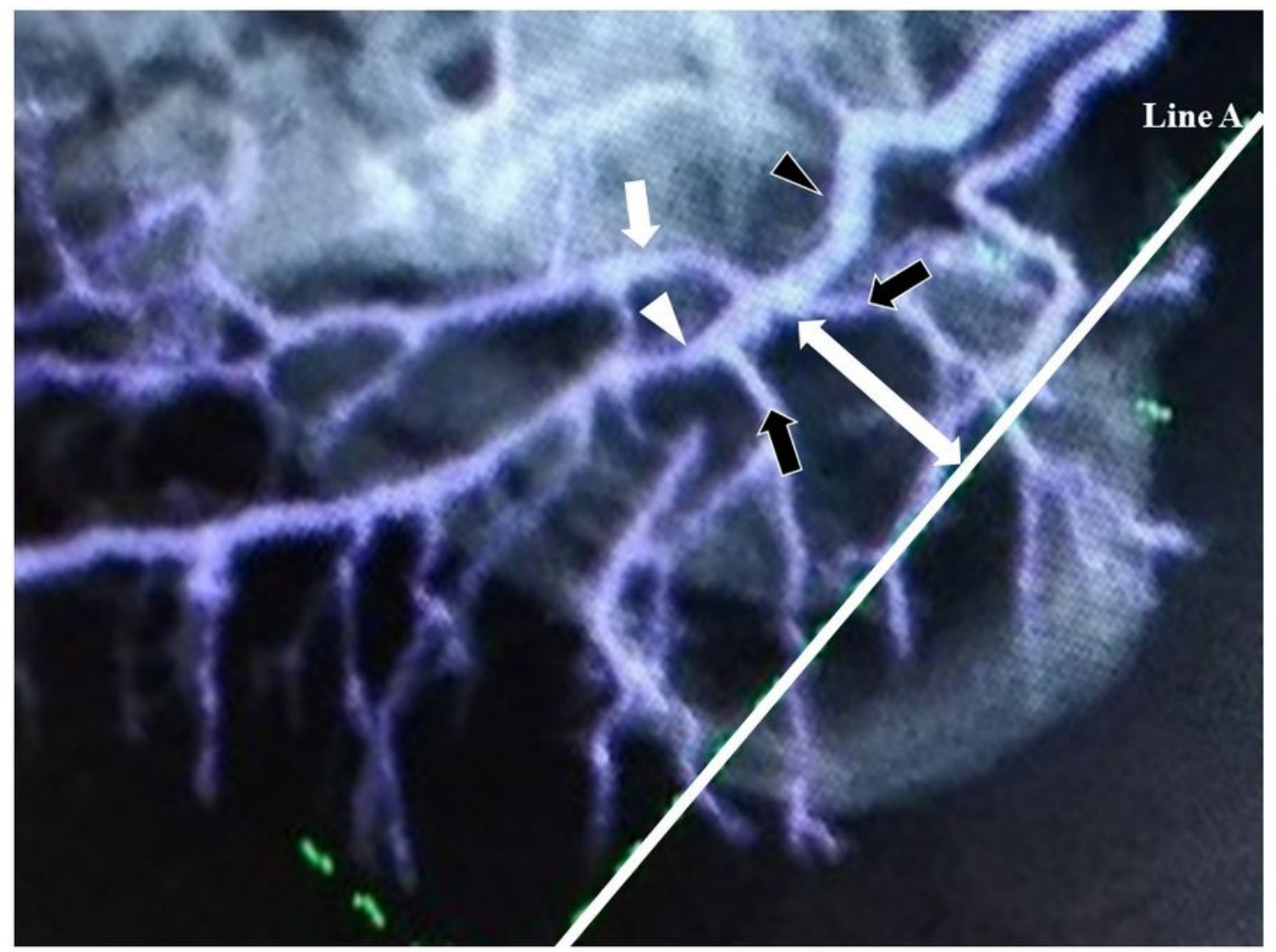

Page 6/8 


\section{Figure 5}

Enhanced computed tomography image showing that the point where the perpendicular distance from line A to the lateral plantar artery is closest is the trifurcation of the lateral plantar artery, medial plantar artery, and one of the medial calcaneal branches. The black arrowhead, white arrowhead, black arrow, and white arrow indicate the posterior tibial artery, lateral plantar artery, medial calcaneal branches (arteries), and medial plantar artery, respectively.

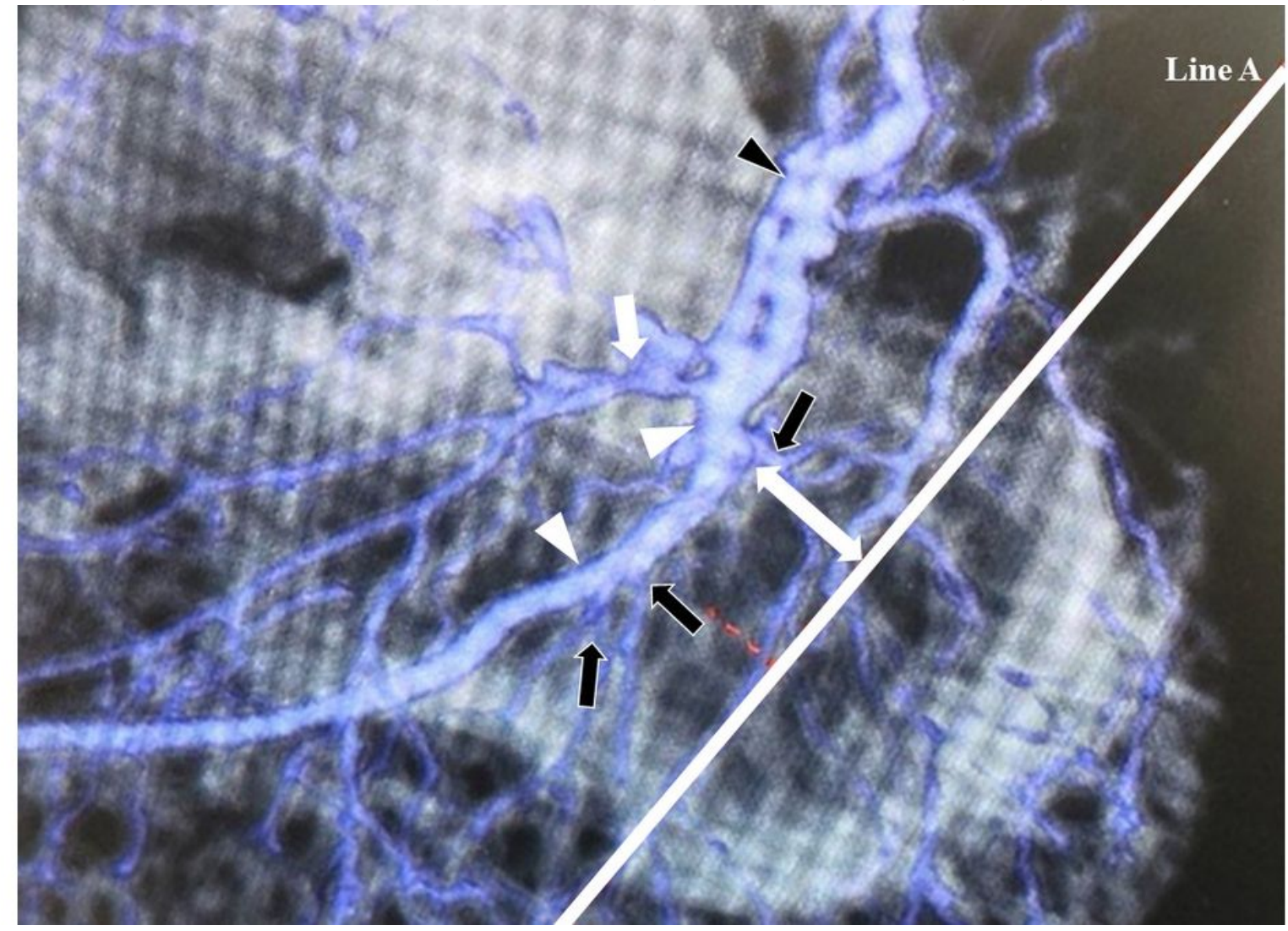

\section{Figure 7}

Enhanced computed tomography image showing a case in which the closest perpendicular distance from the lateral plantar artery to line A was small. The black arrowhead, white arrowhead, black arrow, and white arrow indicate the posterior tibial artery, lateral plantar artery, medial calcaneal branches (arteries), and medial plantar artery, respectively. 


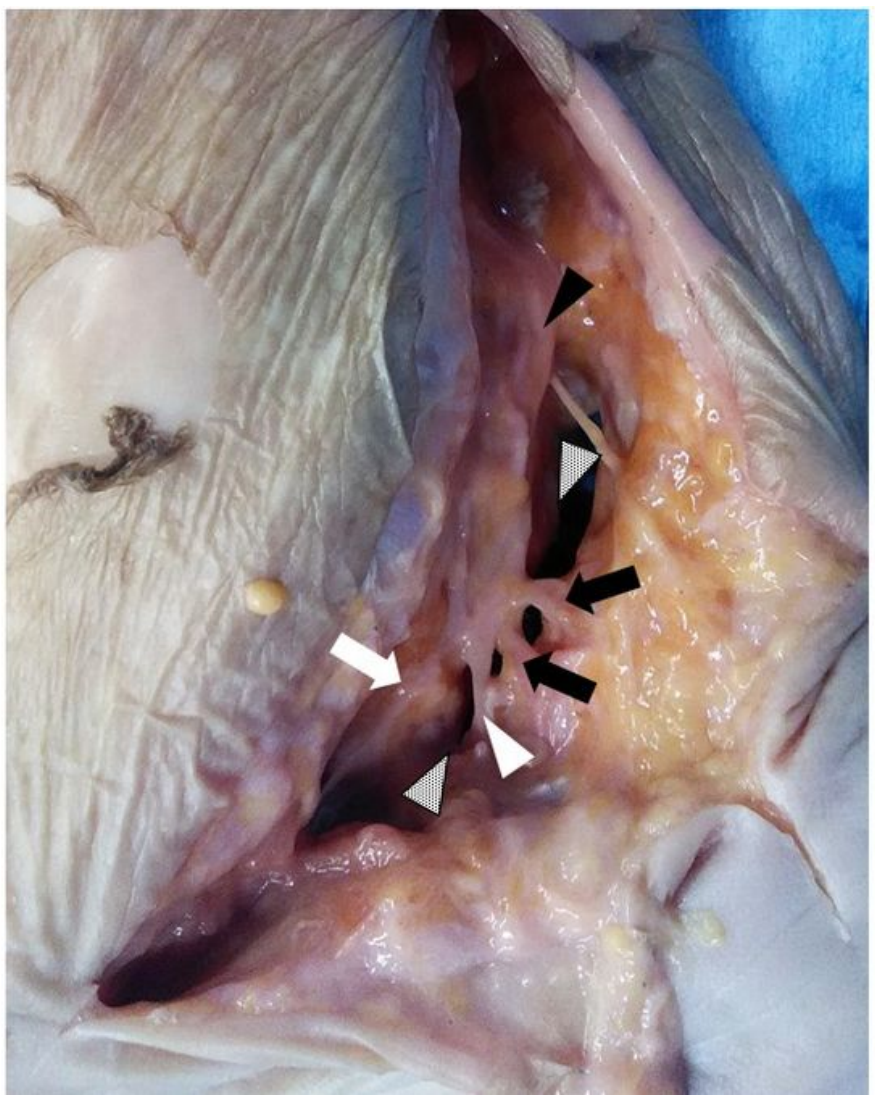

\section{Figure 9}

Photograph showing the dissected posterior tibial artery, lateral plantar artery, and medial plantar artery from the medial aspect. The tibial nerve, medial plantar nerve, lateral plantar nerve, and medial calcaneal nerve branches were dissected. The photograph shows a case in which the closest perpendicular distance from the lateral plantar artery to line A was small after Myerson osteotomy. The Myerson osteotomy line crossed the lateral plantar artery. The dotted arrowhead, black arrowhead, white arrowhead, black arrow, and white arrow indicate the Myerson osteotomy line, posterior tibial artery, lateral plantar artery, medial calcaneal branches (arteries), and medial plantar artery, respectively.

\section{Supplementary Files}

This is a list of supplementary files associated with this preprint. Click to download.

- SupplementalTable1.LPAJFAR.docx

- SupplementalTable1.LPAJFAR.docx 